\title{
A Note on the Optimal Addition of Abscissas to Quadrature Formulas of Gauss and Lobatto Type
}

\author{
By Robert Piessens and Maria Branders
}

\begin{abstract}
An improved method for the optimal addition of abscissas to quadrature formulas of Gauss and Lobatto type is given.
\end{abstract}

1. Introduction. We consider the quadrature formula

$$
\int_{-1}^{+1} f(x) d x \simeq \sum_{k=1}^{N} \alpha_{k} f\left(x_{k}\right)+\sum_{k=1}^{N+1} \beta_{k} f\left(\xi_{k}\right)
$$

where the $x_{k}$ 's are the abscissas of the $N$-point Gaussian quadrature formula. We want to determine the additional abscissas $\xi_{k}$ and the weights $\alpha_{k}$ and $\beta_{k}$ so that the degree of exactness of (1) is maximal. This problem has already been discussed by Kronrod [1] and Patterson [2] and it is well known that the abscissas $\xi_{k}$ must be the zeros of the polynomial $\phi_{N+1}(x)$ which satisfies

$$
\int_{-1}^{+1} P_{N}(x) \phi_{N+1}(x) x^{k} d x=0, \quad k=0,1, \cdots, N,
$$

where $P_{N}(x)$ is the Legendre polynomial of degree $N$. Thus, $\phi_{N+1}(x)$ must be an orthogonal polynomial with respect to the weight function $P_{N}(x)$. Then, the weights $\alpha_{k}$ and $\beta_{k}$ can be determined so that the degree of exactness of (1) is $3 N+1$ if $N$ is even and $3 N+2$ if $N$ is odd.

Szegö [3] proved that the zeros of $\phi_{N+1}(x)$ and $P_{N}(x)$ are distinct and alternate on the interval $[-1,+1]$. Kronrod [1] gave a simple method for the computation of the coefficients of $\phi_{N+1}(x)$. This method requires the solution of a triangular system of linear equations, which is, unfortunately, very ill-conditioned. Patterson [2] expanded $\phi_{N+1}(x)$ in terms of Legendre polynomials. The coefficients of this expansion satisfy a linear system of equations which is well-conditioned, although its construction requires a certain amount of computing time.

The present note proposes the expansion of $\phi_{N+1}(x)$ in a series of Chebyshev polynomials. We also give explicit formulas for the weights $\alpha_{k}$ and $\beta_{k}$. Finally, we consider the optimal addition of abscissas to Lobatto rules. As compared with Patterson's method, our method has three advantages:

(i) It leads to a considerable saving in computing time since the formulas are much simpler.

(ii) The loss of significant figures through cancellation and round-off is slightly reduced, as we verified experimentally. This is in agreement with some theoretical results given by Gautschi [4].

(iii) It is applicable for every value of $N$, while Patterson's method fails in the

Received May 15, 1972, revised March 2, 1973.

AMS (MOS) subject classifications (1970). Primary 65D30; Secondary 33A65. 
Lobatto case for $N=7,9,17,22,27,35,36,37,40, \cdots$, since some of the denominators in his recurrence formulae become zero.

2. Optimal Addition of Abscissas to Gaussian Quadrature Formulas. It is evident that $\phi_{N+1}(x)$ is an odd or even function depending on whether $N$ is even or odd. Thus, $\phi_{N+1}(x)$ can be expressed as

$$
\phi_{N+1}(x)=\sum_{k=0}^{m} b_{k} T_{2 k}(x), \quad \text { if } N \text { is odd, }
$$

and

$$
\phi_{N+1}(x)=\sum_{k=0}^{m} b_{k} T_{2 k+1}(x), \quad \text { if } N \text { is even, }
$$

where $m=[(N+1) / 2]$.

It is clear that the polynomial $\phi_{N+1}(x)$ is only defined to within an arbitrary multiplicative constant. For the sake of convenience, we assume $b_{m}=1$.

From (2), we derive the condition

$$
\int_{-1}^{+1} P_{N}(x) \phi_{N+1}(x) T_{k}(x) d x=0, \quad k=0,1, \cdots, N .
$$

In order to calculate the coefficients $b_{k}, k=0,1, \cdots, m-1,(3)$ or (4) is substituted in (5). This leads to the system of equations

$$
\begin{aligned}
& b_{m-1}=\tau_{1}-1, \\
& b_{m-k}=\sum_{j=1}^{k-1} b_{m-k+j} \tau_{i}+\tau_{k}, \quad k=2,3, \cdots, m,
\end{aligned}
$$

where

$$
\tau_{k}=-\int_{-1}^{+1} P_{N}(x) T_{N+2 k}(x) d x / \int_{-1}^{+1} P_{N}(x) T_{N}(x) d x .
$$

In order to derive a recurrence formula for $\tau_{k}$, we consider the integral

$$
J=\int_{-1}^{+1}\left[x P_{N}(x)-P_{N+1}(x)\right] T_{l}(x) d x .
$$

Using a well-known property of the Chebyshev polynomials, we obtain

$$
J=\frac{1}{2} \int_{-1}^{+1}\left[x P_{N}-P_{N+1}\right] d\left(\frac{T_{l+1}}{l+1}-\frac{T_{l-1}}{l-1}\right),
$$

and, by integrating by parts, this integral can be expressed as

$$
J=\frac{N}{2(l+1)} I_{N, l+1}-\frac{N}{2(l-1)} I_{N, l-1},
$$

where

$$
I_{N, l}=\int_{-1}^{+1} P_{N}(x) T_{l}(x) d x
$$


On the other hand, using a property of the Legendre polynomials, (8) can be transformed into

$$
J=\frac{1}{N+1} \int_{-1}^{+1}\left(1-x^{2}\right) T_{l}(x) d\left(P_{N}(x)\right)
$$

which can be expressed as

$$
J=\frac{2+l}{2(N+1)} I_{N, l+1}+\frac{2-l}{2(N+1)} I_{N, l-1} .
$$

Since $\tau_{k}=I_{N, N+2 k} / I_{N, N}$, the recurrence formula

$$
\tau_{k+1}=\frac{[(N+2 k-1)(N+2 k)-(N+1) N](N+2 k+2)}{[(N+2 k+3)(N+2 k+2)-(N+1) N](N+2 k)} \tau_{k},
$$

where $\tau_{1}=(N+2) /(2 N+3)$ can be easily derived from (10) and (12).

System (6) is easier to construct than the corresponding system of Patterson [2], inasmuch as his method requires a set of recursions of variable lengths, while in our method only one recursion is needed. Moreover, further economy is achieved in solving the equation $\phi_{N+1}(x)=0$, since, using a modification of Clenshaw's algorithm of summation, an odd or even Chebyshev series can be evaluated more efficiently than an odd or even Legendre series [5, p. 10]. Indeed, the computing time can be halved.

Explicit formulas for the weights are

$$
\begin{array}{ll}
\alpha_{k}=\frac{C_{N}}{P_{N}^{\prime}\left(x_{k}\right) \phi_{N+1}\left(x_{k}\right)}+\frac{2}{N P_{N-1}\left(x_{k}\right) P_{N}^{\prime}\left(x_{k}\right),}, & k=1,2, \cdots, N, \\
\beta_{k}=\frac{C_{N}}{\phi_{N+1}^{\prime}\left(\xi_{k}\right) P_{N}\left(\xi_{k}\right)}, & k=1,2, \cdots, N+1,
\end{array}
$$

where $C_{N}=2^{2 N+1}(N !)^{2} /(2 N+1) !$.

3. Optimal Addition of Abscissas to Lobatto Quadrature Formulas. We now consider the quadrature formula

$$
\int_{-1}^{+1} f(x) d x \simeq \sum_{k=0}^{N+1} \alpha_{k} f\left(x_{k}\right)+\sum_{k=1}^{N+1} \beta_{k} f\left(\xi_{k}\right)
$$

where the $x_{k}$ 's are abscissas of the Lobatto quadrature formula. Consequently, $x_{0}=-1, x_{N+1}=+1$ and $x_{1}, x_{2}, \cdots, x_{N}$ are the zeros of the Jacobi polynomial $P_{N}{ }^{(1,1)}(x)$. It is our purpose to determine the free abscissas $\xi_{k}$ and the weights $\alpha_{k}$ and $\beta_{k}$ so that the degree of exactness of (16) is maximal. Then, $\xi_{k}$ must be a zero of the polynomial $\phi_{N+1}(x)$ which satisfies

$$
\int_{-1}^{+1}\left(1-x^{2}\right) P_{N}^{(1,1)}(x) \phi_{N+1}(x) T_{k}(x) d x=0, \quad k=0,1,2, \cdots, N .
$$

Again, we express $\phi_{N+1}(x)$ in terms of Chebyshev polynomials as in (3) or (4), according to the parity of $N$. The coefficients $b_{k}$ can be found by solving the system (6) where

$$
\tau_{k}=-\int_{-1}^{+1}\left(1-x^{2}\right) P_{N}^{(1,1)} T_{N+2 k} d x / \int_{-1}^{+1}\left(1-x^{2}\right) P_{N}^{(1,1)} T_{N} d x
$$


Using the relation

$$
\int_{-1}^{+1}\left(1-x^{2}\right) P_{N}^{(1,1)} T_{l} d x=\frac{1}{N+2}\left[(l+2) I_{N+1, l+1}-(l-2) I_{N+1, l-1}\right],
$$

where $I_{N . l}$ is defined by (11), the recurrence formula

$$
\tau_{k+1}=\frac{[(N+2 k-1)(N+2 k-2)-(N+1)(N+2)](N+2 k+2)}{[(N+2 k+3)(N+2 k+4)-(N+1)(N+2)](N+2 k)} \tau_{k}
$$

can be derived from (13).

The starting value for (19) is

$$
\tau_{1}=3(N+2) /(2 N+5) \text {. }
$$

The expressions for the weights are

$$
\alpha_{k}=\frac{C_{N}}{2 P_{N}^{\prime}\left(x_{k}\right) \phi_{N+1}\left(x_{k}\right)}+\frac{2}{(N+1)(N+2)\left[P_{N+1}\left(x_{k}\right)\right]^{2}},
$$

for $k=1,2, \cdots, N$,

where $C_{N}=2^{2 N+3}[(N+1) !]^{2} /(2 N+3) !$.

Appendix. Computer program. In this appendix, we describe a FORTRAN program for the construction of the quadrature formula (1). A listing of this program is reproduced in the supplement at the end of this issue. A program for the construction of the quadrature formula (11) may be obtained from the authors.

The program consists of three subroutines: the main subroutine KRONRO and two auxiliary subroutines ABWE1 and ABWE2, which are called by KRONRO.

In KRONRO the coefficients of the polynomial $\phi_{N+1}(x)$ are calculated.

In ABWE1 the abscissas $x_{k}$ and weights $\alpha_{k}$ are calculated.

In ABWE2 the abscissas $\xi_{k}$ and weights $\beta_{k}$ are calculated.

The abscissas are calculated using Newton-Raphson's method. Starting values for this iterative process are provided by [6]

$$
x_{k} \simeq\left(1-\frac{1}{8 N^{2}}+\frac{1}{8 N^{3}}\right) \cos \left(\frac{2 k-1 / 2}{2 N+1} \pi\right)
$$

and

$$
\xi_{k} \simeq\left(1-\frac{1}{8 N^{2}}+\frac{1}{8 N^{3}}\right) \cos \left(\frac{2 k-3 / 2}{2 N+1} \pi\right) .
$$

The program has been tested on the computer IBM 370/155 of the Compuiting Centre of the University of Leuven, for $N=2(1) 50(10) 200$. The computations were carried out in double precision (approximately 16 significant figures). For $N=200$, the maximal absolute error of the abscissas is $8.6 \times 10^{-16}$ and of the weights $3.3 \times 10^{-15}$. 
For $N=50$, the computing time is $1.7 \mathrm{sec}$, for $N=100,6.4 \mathrm{sec}$. and for $N=200$, $24.7 \mathrm{sec}$.

Acknowledgement. This research was supported in part by the Fonds voor Kollektief Fundamenteel Onderzoek under Grant No. 10.174.

Applied Mathematics Division

Katholieke Universiteit Leuven

Heverlee, B-3030, Belgium

1. A. S. Kronrod, Nodes and Weights for Quadrature Formulas, "Nauka", Moscow, 1964; English transl., Consultants Bureau, New York, 1965. MR 32 \#597; \#598.

2. T. N. L. PATTERSON, "The optimum addition of points to quadrature formulae," Math. Comp., v. 22, 1968, pp. 847-856. MR 39 \#3701.

3. G. SZEGö, "Uber gewisse orthogonale Polynome, die zu einer oszillierenden Belegungsfunktion gehören," Math. Ann., v. 110, 1935, pp. 501-513.

4. W. GAUTSCHI, "On the construction of Gaussian quadrature rules from modified moments," Math. Comp., v. 24, 1970, pp. 245-260. MR 44 \#2341a.

5. C. W. Clenshaw, Chebyshev Series for Mathematical Functions, National Physical Laboratory Mathematical Tables, vol. 5, Her Majesty's Stationary Office, London, 1962. MR 26 \#362.

6. F. G. TRIComI, Vorlesungen über Orthogonalreihen, Die Grundlehren der math. Wissenschaften in Einzeldarstellungen mit besonderer Berücksichtigung der Anwendungsgebiete, Band 76, Springer-Verlag, Berlin, 1955. MR 17, 30. 
SUPPLEMENT TO

A Note on the Optimal Addition of Abscissas to Quadrature Formulas of Gauss and Lobatto Type

by

ROBERT PIESSENS \& MARIA BRANDERS

pp. 135-139, this issue

SLERCLTINE KRONRC $(A, A, h 1, h 2, E P S$, IER )

C

THIS SLERCLTINE CALCLLATES THE ABSCISSAS A ANC WEIGHTS WI CF THE (2*A+1)-PCINT GLACRATLRE FORMULA WHICF IS OBTAIAEC FRCN THE A-PCINT GALSSIAN RULE BY OPTINAL ACCITION OF A+1 PCINTS. THE CPTIMALLY ACCED POINTS ARE CALLEC KRCNROC ABSCISSAS. AESCISSAS ANC hEIGHTS ARE CALCULATEC FCR INTEGRATICA CN THE IATERVAL (-1,1). SIACE THIS OUACRATURE FCRMLLA IS SYNMETRICAL WITH RESPECT TO THE CRIGINE,CNLY THE ACANEGATIVE ABSCISSAS ARE CALCULATEC. WEIGHTS CORRESPCNCING TC SYMMETRICAL ABSCISSAS ARE EGLAL.

IA ACCITION, THE WEIGFTS hZ CF THE GAUSSIAA RULE ARE CALCLLATEC.

$R E A L * E A, A K, A N, B, C, T A L, h 1, h 2, X X$

[INEASICN A(2C1), E(201), TAU (201),WI(201),h2(2C1)

CCNMCA C, INDEKS

\section{IAPLTTPARAMETERS}

A CRCER CF THE GALSSIAA CUACRATURE FCRNULA TO HHICH AESCISSAS NUST BE ACLEC.

EPS REGLESTED ABSCLLTE ACCURACY OF THE ABSCISSAS. THE ITERATIVE PRCCESS TERMIAATES IF THE AESCLUTE CIFFERENCE BETHEEA THO SUCCESSIVE APPRCXIMATIONS IS LESS THAN EPS.

CLTPLTFARANETERS

A VECTCR OF CINEASICA $\Lambda+1$ WHICH CCATAINS THE NONNEGATIVE $\triangle B S C I S S A S . A(1)$ IS THE LARGEST AESCISSA.A(2EK) IS A GAUSSIAA ABSCISSA.A(2:K-1) IS A KRCNRCC ABSCISSA.

W 1 VECTCR CF DINEASICA $\Lambda+1$ WHICH CCATAINS THE WEIGHTS CCRRESPONDING TC THE ABSCISSAS A.

h2 VECTCR OF CINEASICA $A+1$, CONTAININE THE GAUSSIAN hEICHTS. W2(2*K-1) $=C$ AND W2(2*K) IS THE GALSSIAN hEIGHT CCRRESPCACIAE TC A(2*K).

IER ERRCR COCE IF IER=0 ALL AESCISSAS ARE FCUNC TC WITHIN THE REQLESTED ACCLRACY. IF IER=1 ONE CF THE ABSCISSAS IS NCT FCLNC AFTER 5C ITERATICN STEPS ANC THE CCMPLTATIOA IS TERPINATEC.

REGLIREC SLBPROGRANS

ABHEI CALCULATES THE KRCARCD ABSCISSAS ANC CCRRESPONCING hEIGHTS.

ABhE2 CALCULATES THE GALSSIAN ABSCISSAS ANC THE CCRRESPONDING hEIGHTS. 
C

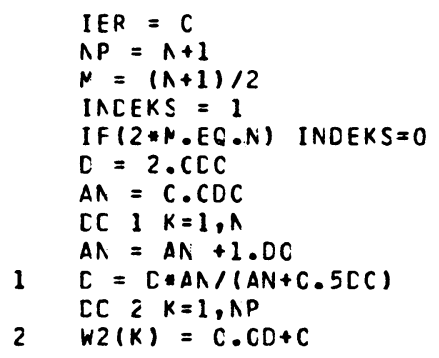




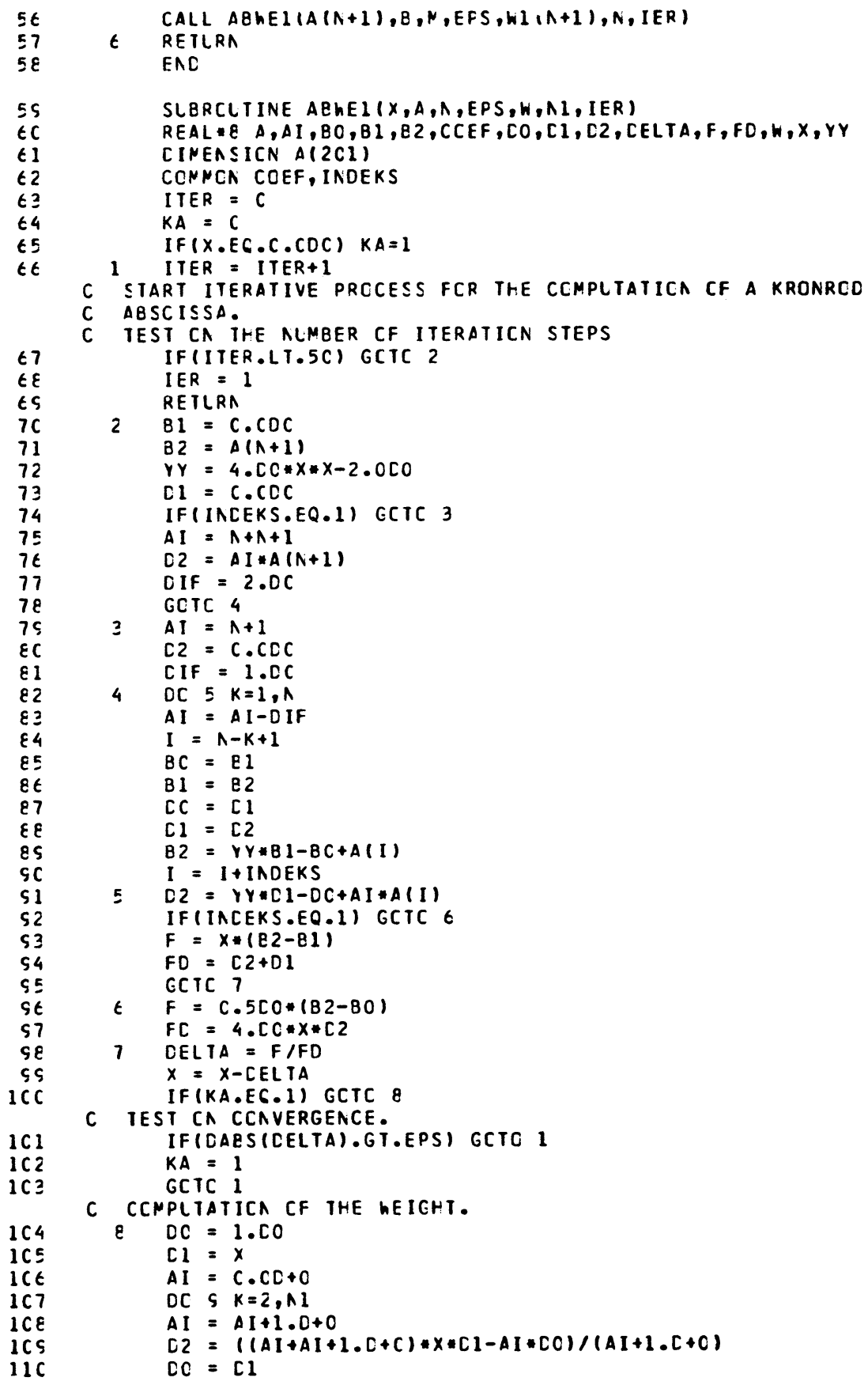




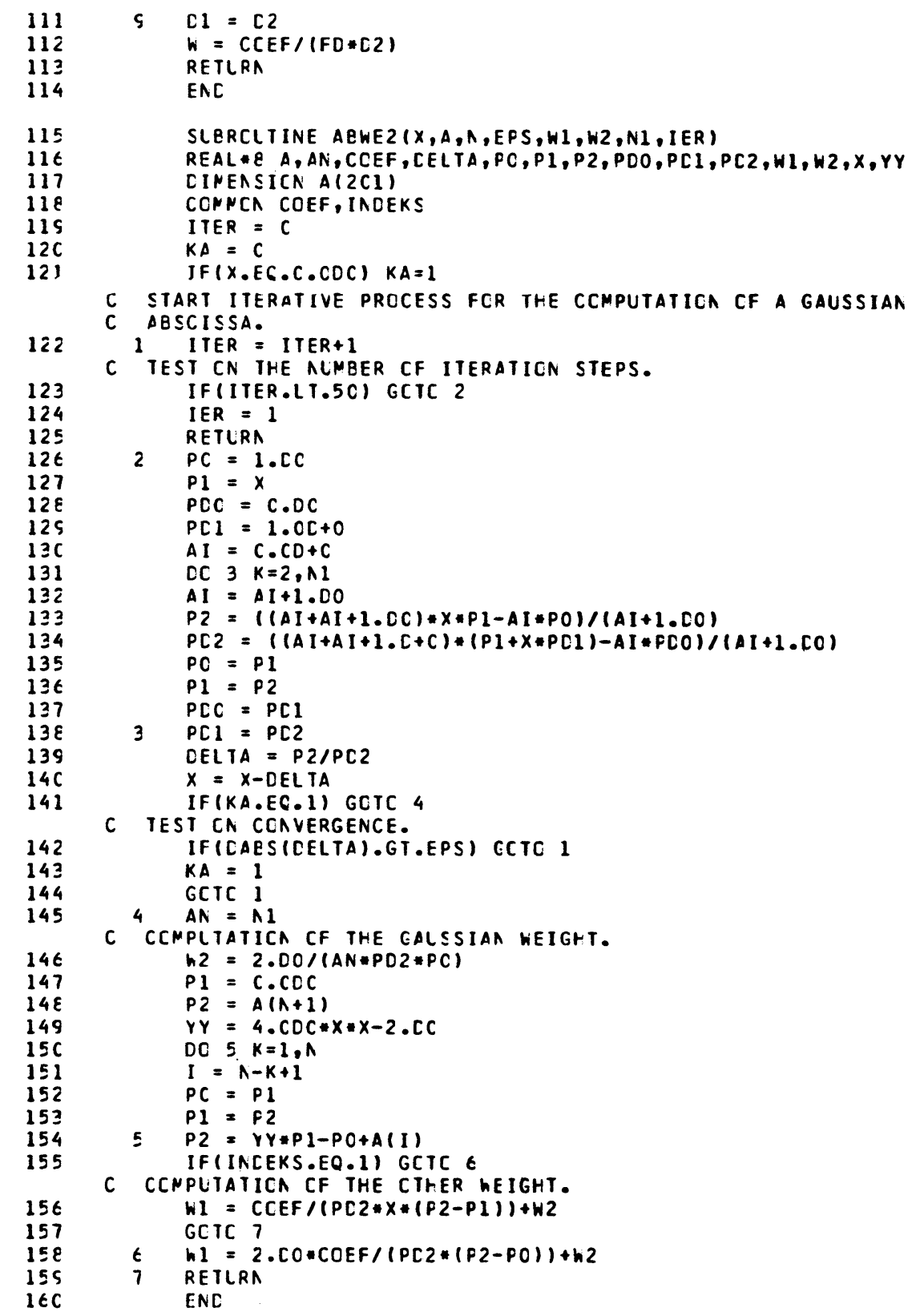

